\title{
Non-invasive removal of self-locking entrapped nephrostomy tubes
}

\author{
Fabio Campodonico ${ }^{1}$, Umberto Geremia Rossi ${ }^{2}$, Marco Ennas ${ }^{1}$, Alessandro Valdata ${ }^{2}$, \\ Antonia Di Domenico ${ }^{1}$, Francesco Pinna ${ }^{2}$, Carlo Introini ${ }^{1}$ \\ ${ }^{1}$ Urology Unit, Ospedale Galliera, Genova, Italy; \\ ${ }^{2}$ Interventional Radiology Unit, Ospedale Galliera, Genova Italy.
}

\begin{abstract}
Summary Introduction: The removal of an encrusted nephrostomy tube can be a challenging maneuver. Urological literature is very bare in detailing techniques for removal of entrapped percutaneous catheters. We present a simple, safe and non-invasive technique of nephrostomy removal using a vascular introducer sheath, useful to manage complicated situations such as nephrostomies blocked for severe encrustations or disabled in their self-locking system. Surgical technique: The nephrostomy tube is cut and the stump is passed with a suture needle. The suture is passed through the inner vascular introducer sheath tip, and the introducer is then removed. The introducer sheath is advanced over the nephrostomy until joining the pigtail segment, under fluoroscopy guidance. Thus the suture is pulled out with strenght to contrast the opposite stiffness of the encrusted coil, until the nephrostomy has safely come out.

Comment: The sheath exchange technique is quick, involves less manipulation through the perirenal fascia and kidney, and is suitable for different conditions of entrapped nephrostomies.
\end{abstract}

KEY WORDS: Nephrostomy tube; Encrustation; Vascular sheath; Percutaneous nephrostomy.

Submitted 2 March 2020; Accepted 15 March 2020

\section{INTRODUCTION}

Nephrostomy tube is a common device used in several urological procedures during urgent or elective surgery. However, a number of complications can occur to patients such as urinary infections, bleeding, nephrostomy malfunction due to displacement or tube encrustation. Moreover, the calcified tube disables the release system in case of a self-locking nephrostomy. Thus, the removal of an encrusted nephrostomy tube can be a challenging maneuver. Urological literature is very bare in detailing techniques for removal entrapped percutaneous catheters (1). We present a simple, non-invasive technique of nephrostomy removal, useful to manage complicated situations.

\section{NOTES OF SURGICAL TECHNIQUE}

When nephrostomy tube substitution is impossible for severe encrustation, firstly a guidewire should be used tapping the tip through the tube, under fluoroscopy guidance. When the tube patency is not achieved, the rigid stump of the guidewire can be used in the same way. However, in case of a self-locking nephrostomy tube, the use of guidewire is worthless and the release system could be enabled by encrustations. In both conditions, a non-invasive technique of nephrostomy removal is possible using a safety technique. As a consequence of an 8 French malfunctioning nephrostomy, a vascular introducer sheath of 9 French diameter and 10 $\mathrm{cm}$ long (Radifocus ${ }^{\circledR}$ Introducer II, Terumo Europe, Leuven, Belgium) is used with the following steps. The stopcock of the tube is cut and the stump is passed with a needle of a 2/0 silk suture (Figure 1A). The silk suture is passed through the inner vascular introducer sheath tip (Figure 1B), and the introducer is then removed (Figure 1C). The introducer sheath is advanced over the nephrostomy by threading the suture through the tube in order to maintain a straight position (Figure 1D).

The sheath is advanced progressively until joining the pigtail segment (Figure 1E), under fluoroscopy guidance, thus the suture is pulled out with strenght to contrast the opposite stiffness of the encrusted coil, until the nephrostomy is removed (Figure 1F). Once the encrusted nephrostomy is removed, the introducer sheath may be left in place acting as a guide for a new nephrostomy tube. Radiologic steps are summarized in Figure 2.

\section{Discussion}

A few percutaneous techniques have been proposed in non-urological literature. Canby-Hagino et al. reported the intraluminal pneumatic lithotripsy. The lithotripsy probe is directly inserted through the nephrostomy encrusted lumen, acting like a mini-jackhammer (2). A similar technique was reported by Mangera et al. by using a 1-mm Lithoclast probe. The lithotripsy is gently carried on by pulling the nephrostomy onto the Lithoclast. An intra and extraluminal fragmentation is done along the straight portion of the tube, under radiological control, to avoid a dangerous exit of the Lithoclast probe into the kidney (3). Baron and McClennan firstly reported the simple exchange of catheters using an angiographic catheter sheath. They suggest to leave the sheath on the nephrostomy next to the stopcock of the new pigtail catheter (4). Pollack reported on two methods to remove an encrusted nephrostomy.

The first permits to remove a tube encrusted along the external tract, but patent in transparietal and coiled segments. The second is similar to our technique, based on 


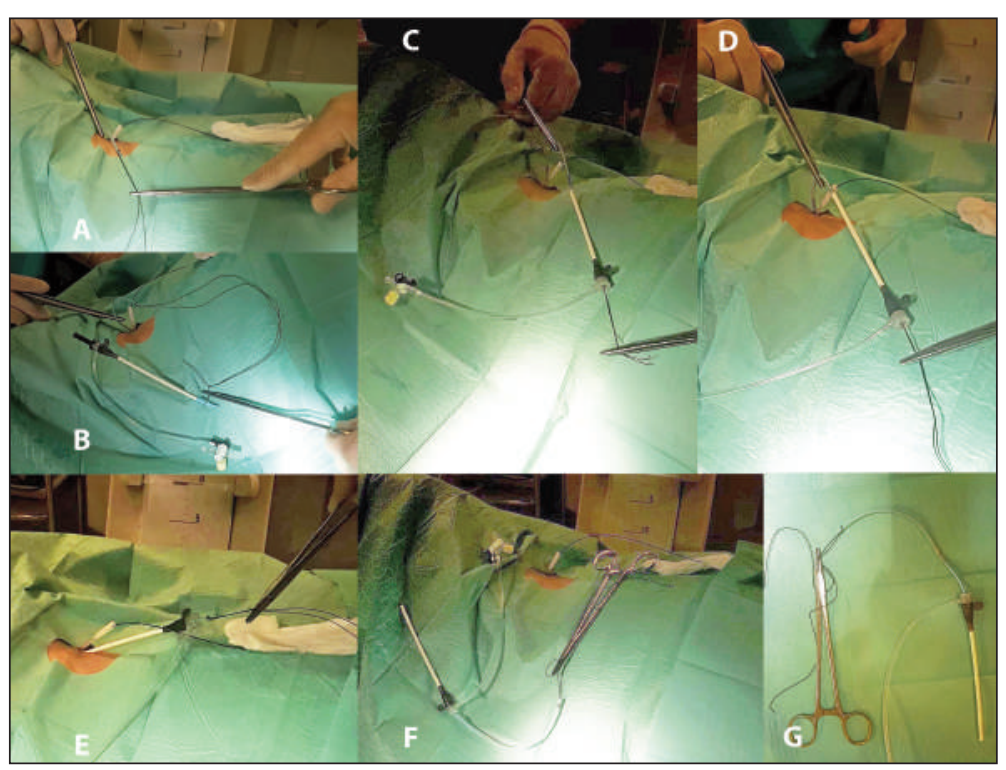

Figure 1.

Steps of removal of entrapped nephrostomy.

A) The stopcock of the tube is cut and the stump is passed with a needle of a 2/0 silk suture;

B) The silk suture is passed through the inner vascular introducer sheath tip;

C) The introducer is removed;

D) The introducer sheath is advanced over the nephrostomy by threading the suture through the tube;

E) The sheath is advanced until joining the pigtail segment;

F) The suture is pulled out with strenght until the nephrostomy is removed;

G) The nephrostomy removed.

Figure 2.

Radiological steps.

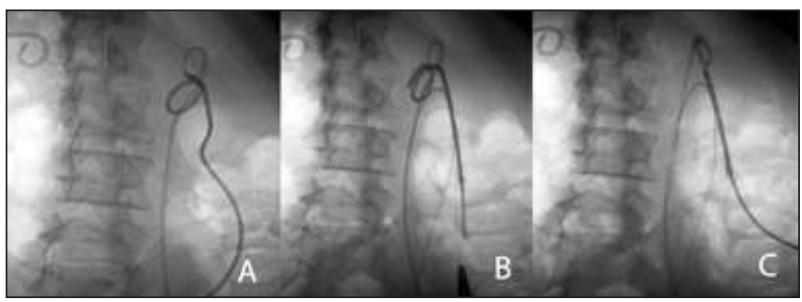

the use of a vascular sheath (5). Farooq et al. depicted in details the technique employing the angiographic catheter sheath, however in an interventional radiology context (6). Finally, studies using a similar technique are very few (46 ), and mainly refer to the expertise of interventional radiologists in materials and techniques they use for percutaneous retrieval of intravascular foreign bodies (7). Our report, however, has two points to be highlighted: this is the first description appearing in urological literature and the nephrostomy tubes we used was a self-locking type, thus resulting in a complicated condition.

The technique depicted showed to be useful, simple and safe in the most difficult cases of occluded and entrapped nephrostomy tubes. As reported by Baron, "the sheath exchange method is quicker and involves less manipulation through the perirenal fascia and kidney, less fluoroscopy time, and ensures preservation on the tract for direct insertion of the new nephrostomy tube". When non-invasive maneuvers of nephrostomy removal fail, choice of treatments can be extracorporeal shock wave lithotripsy, ureteroscopy, percutaneous or open surgery.

The management of nephrostomy tubes is a common urological task. The urologist should be confident with the different problems encountered, and the technique reported could implement the practical expertise.

\section{Aknowledgement}

We wish to thank Carlo Oliveri and Lino Squillace for technical assistance.

\section{References}

1. Alnadhari I, Alwan MA, Salah MA, Ghilan AM. Treatment of retained encrusted ureteral Double-J stent. Arch Ital Urol Androl. 2019; 18; 90:265-269.

2. Canby-Hagino ED, Caballero RD, Harmon WJ. Intraluminal pneumatic lithotripsy for the removal of encrusted urinary catheters. J Urol. 1999; 162:2058-60.

3. Mangera A, Mehta S, Hastie KJ, et al. Lithoclast removal of an encrusted nephrostomy tube. Ann R Coll Surg Engl. 2010; 92:353-4.

4. Baron RL, McClennan BL. Replacing the occluded percutaneous catheter. Radiology. 1981; 141:824.

5. Pollack Hl, Banner MP. Replacing blocked or dislodged percutaneous nephrostomy and ureteral stent catheters. Radiology. 1982; 145:203-5.

6. Farooq A, Agarwal S, Vaughan J. Safe removal of an ecrusted nephrostomy tube using a vascular sheath: a technique revisited. Cardiovasc Intervent Radiol. 2013; 36:820-3.

7. Rossi UG, Rollandi GA, Ierardi AM, et al. Materials and techniques for percutaneous retrieval of intravascular foreign bodies. $J$ Vasc Access. 2019; 20:87-94.

\section{Correspondence}

Fabio Campodonico, MD (Corresponding Author)

fabio.campodonico@galliera.it

Marco Ennas, MD

marco.ennas@galliera.it

Antonia Di Domenico, MD

antonia.didomenico@galliera.it

Carlo Introini, MD

carlo.introini@galliera.it

Urology Unit, Ospedale Galliera

Via Volta 8, 16128 Genova (Italy)

Alessandro Valdata, MD alessandro.valdata@galliera.it

Umberto Geremia Rossi, MD umberto.rossi@galliera.it

Francesco Pinna, MD

francesco.pinna@galliera.it

Interventional Radiology Unit, Ospedale Galliera

Via Volta 8, 16128 Genova (Italy) 